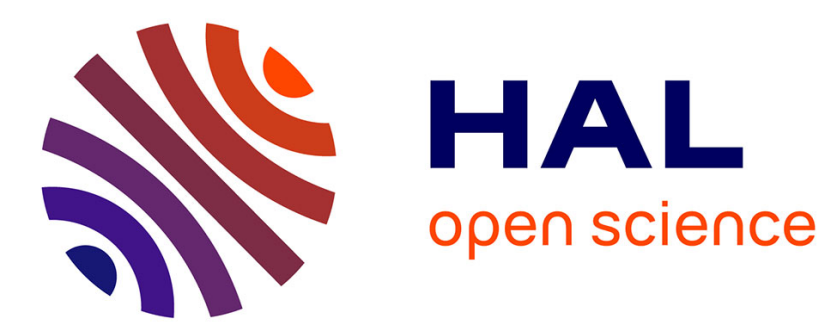

\title{
Enveloppe psychique et Moi-peau dans la prise en charge groupale de patients psychotiques
}

\author{
Clarisse Vollon, Guy Gimenez
}

\section{To cite this version:}

Clarisse Vollon, Guy Gimenez. Enveloppe psychique et Moi-peau dans la prise en charge groupale de patients psychotiques. Psychotherapies, 2015, Transitions, 36, pp.259 - 265. 10.3917/psys.154.0259 . hal-01375574

\section{HAL Id: hal-01375574 \\ https://hal.science/hal-01375574}

Submitted on 26 Oct 2016

HAL is a multi-disciplinary open access archive for the deposit and dissemination of scientific research documents, whether they are published or not. The documents may come from teaching and research institutions in France or abroad, or from public or private research centers.
L'archive ouverte pluridisciplinaire HAL, est destinée au dépôt et à la diffusion de documents scientifiques de niveau recherche, publiés ou non, émanant des établissements d'enseignement et de recherche français ou étrangers, des laboratoires publics ou privés. 


\title{
Enveloppe psychique et Moi-peau dans la prise en charge groupale de patients psychotiques
}

\author{
Clarisse Vollon ${ }^{1}$ et Guy Gimenez ${ }^{2}$
}

\section{Résumé}

Notre article a pour objectif de questionner et de redéfinir les notions de Moi-peau et d'enveloppe(s) psychique(s). Cette démarche théorique, en tant que travail préliminaire, vise à introduire une étude plus globale sur la qualité et la dynamique des enveloppes psychiques que se soit dans le cadre de prises en charge psychothérapeutiques individuelles et/ou groupales. Notre méthode consiste à revenir sur les différents apports épistémologiques, notamment ceux issus du corpus freudien afin d'apporter un éclairage différent sur les notions de Moi-peau et d'enveloppes psychiques. En effet, elles entretiennent dans la littérature une forte interdépendance. Cette démarche va nous permettre progressivement d'observer qu'il est non seulement possible d'appréhender la notion d'enveloppe psychique comme une refonte de la notion de Moi-peau mais qu'il existe plusieurs types de Moi-peau et d'enveloppes psychiques que nous nous proposons ici de répertorier pour la première fois et que nous proposons d'organiser en six catégories spécifiques.

ouvent nous pouvons entendre de la part des patients dans les groupes thérapeutiques que nous menons: "Est-ce que je peux sortir $d u$ groupe? », ou "Je n'ai pas envie de parler aujourd'hui, ça ne regarde personne", mais aussi "Est-ce que moi aussi je peux venir dans le groupe?»... Problématiques des entrées et des sorties, du dedans et du dehors, du contenant et du contenu: la prise en charge groupale de patients psychotiques mobilise et questionne spécifiquement sur ces thèmes. Ainsi, Falguière (2002), et plus récemment Laugier et Toliou (2009), rappellent que les patients psychotiques peuvent exprimer en situation de groupe des difficultés à appréhender leurs limites dans la relation. Ces dispositifs requièrent une attention spécifique ainsi que la formulation d'interventions centrées sur l'ensemble des zones délimitantes et bordantes de l'appareil psychique groupal (Kaës, 1979) et des appareils psychiques individuels de chacun des membres du groupe. Dans cette perspective, le recours aux notions d' «enveloppe psychique» ou de «Moi-peau » (Anzieu, 1985) est éclairant pour formuler ces interventions. Seulement, nous nous sommes aperçus à travers notre expérience clinique, qu'il était nécessaire de les réinterroger spécifiquement dans le cadre de la prise en charge groupale de patients psychotiques: le Moi-peau diffère selon l'organisation psychique d'un sujet (Anzieu, 1987) et la texture de l'enveloppe psychique évolue selon la dynamique d'un groupe (Vollon \& Gimenez, 2014). Qu'entendons nous spécifiquement par Moi-peau et enveloppe psychique dans un groupe thérapeutique de patients

???????????????

??????????????

??????????????????

??????????

2 ???????????????

??????????????

??????????????????

?????????? 
psychotiques? Quelles sont les articulations possibles entre ces deux notions? Comment nous sont-elles accessibles durant les séances et comment pouvons nous nous en saisir pour formuler nos interventions? Pour répondre à ces questions il nous paraît important de revenir dans un premier temps sur les différents apports théoriques qui nous permettent de les appréhender et de les définir. Nous nous attacherons à présenter et analyser dans un second temps les extraits d'une séance d'un groupe psychanalytique de parole mené dans un service de réinsertion et de réhabilitation pour des patients psychotiques particulièrement chroniques.

\section{Aux origines du concept de Moi-peau}

Tout au long de son œuvre, Freud a envisagé topologiquement l'appareil psychique en termes de surfaces séparatrices, filtres et autres espaces différenciés. Ainsi, dès 1895, il avance l'existence d'une «barrière de contact» constituée de deux types de neurones: les perméables qui délimitent les stimuli provenant du monde extérieur, et les imperméables qui les filtrent avec plus ou moins de succès. En 1900, par l'analyse des rêves, il propose également la différenciation de deux systèmes grâce à une censure: l'Inconscient (Ics) qui a pour objectif de déverser l'ensemble des excitations internes et le Préconscient-Conscient (Pc-Cs) qui a pour objectif d'inhiber ce déversement et de les orienter pour pouvoir mieux les écouler à travers l'action motrice. Au-delà d'être envisagée comme une instance séparatrice de deux systèmes, cette censure est aussi garante de la gestion du passage des éléments psychiques entre eux : elle semble alors constituer l'origine épistémologique la plus ancienne du Moi-peau et de l'enveloppe psychique. Mais c'est à partir de 1923, en développant et précisant la définition du Moi, que Freud introduit pour la première fois le terme d'«enveloppe » se construisant à la jonction des systèmes Pc-Cs/Ics: «Le Moi n'enveloppe pas complètement le Ça, mais seulement dans les limites où le systèmes Pc constitue sa surface, donc à peu près comme le disque germinatif est posé sur l'œuf » (Freud, 1923, p. 24). Par la suite, grâce à l'utilisation de l'image du bloc-notes magique, Freud (1925) finit par proposer une conception de l'appareil psychique totalement en feuillets. Il distingue deux couches, une première qui a une fonction d'ins- cription des traces (le papier ciré du bloc-notes) et une fonction de filtre ou pare-excitante (la feuille de celluloïd): «J'ai développé l'idée que l'appareil perceptif psychique comporte deux couches: l'une externe, le pare-stimulus, destiné à réduire la grandeur des excitations qui arrivent du dehors, l'autre, derrière celle-ci, surface réceptrice de stimulus, le système Pc-Cs. » (Freud, 1925, p. 121).

C'est en 1985 qu'Anzieu propose et développe la notion de Moi-peau pour la première fois. Cet élément, qui appartient à la réalité fantasmatique de l'individu, assume huit fonctions dans l'appareil psychique: la contenance psychique, la maintenance psychique, la pare-excitation, l'individuation, l'intersensorialité, le soutien de l'excitation sexuelle, la recharge libidinale, et l'inscription de la trace sensorielle. Issu notamment de l'intériorisation des expériences tactiles, il est intéressant de souligner qu'Anzieu propose également une approche du Moipeau en feuillets, s'inscrivant ainsi dans l'héritage freudien de l'appréhension de l'appareil psychique. Il évoque alors un feuillet externe issu des expériences vécues avec l'«entourage maternant» (tel le papier ciré du bloc-notes) et un feuillet interne qui correspond à «l'enveloppe interne, à la surface du corps du bébé, lieu et instrument d'émission de messages » (Anzieu, 1985, p. 56) à l'instar de la feuille en celluloïd. La conception du Moi-peau et de sa consistance en fonction de l'organisation psychique de l'individu a évolué au fur et à mesure des années s'articulant progressivement avec la notion d'enveloppe. En 1990 Anzieu avance que le Moi-peau se compose d'un Moi-Noyau résultant de l'introjection d'un objet primordial durant les expériences de la vie d'un individu et d'un Moi-enveloppe remplissant plus globalement les fonctions du Moi-peau (Anzieu, 1990, p. 47).

\section{La notion d'Enveloppe psychique: entre pluralité et refonte de la notion de Moi-peau}

La notion d'enveloppe(s) psychique(s) est plus difficile à définir. Utilisée comme synonyme du Moi chez Freud, ou comme partie constituante du Moipeau chez Anzieu, ce dernier finit d'ailleurs par l'envisager en tant qu'enveloppe psychique à part entière (Anzieu, 1990, p. 71). Elle se structure comme le Moi-peau, en deux couches: une externe qui fait 
écran aux stimulations extérieures (enveloppe d'excitation) et une interne qui met en lien les stimulations extérieures et l'environnement intrapsychique (enveloppe de communication). Par ailleurs, nous avons été interpellés par la diversité des modalités et fonctions de l'enveloppe psychique dans la littérature, rendant cette notion plurielle. Nous en avons relevé à ce jour vingt-huit, que nous organisons arbitrairement en six catégories (faute d'un support théorique déjà existant à ce sujet).

Tout d'abord, les enveloppes sensorielles de l'enveloppe psychique. Dès 1923, Freud souligne que le Moi est composé d'une "calotte acoustique», un organe sensoriel lui permettant de percevoir les stimulations extérieures. Cette idée va être reprise par Anzieu (1990) qui propose quatre types d'enveloppes psychiques spécifiques du champ sensoriel: l'enveloppe visuelle, l'enveloppe sonore, l'enveloppe de clarté obscurité et l'enveloppe chromatique.

Ensuite les enveloppes impliquées dans les expériences précoces. L'enveloppe de soin décrite par Brazelton (1982) caractérise la façon dont la mère enveloppe l'enfant de soi pour satisfaire au maximum ses besoins. L'enveloppe habitat (ibid.) correspond à l'acquisition par le bébé de la distinction des besoins corporels et des besoins psychiques. L'enveloppe utérine (Anzieu, 1990) permet l'émergence de la conscience et l'ébauche d'un système perception/conscience. L'enveloppe narcissique (ibid.) favorise la distinction entre Soi et non Soi. L'enveloppe individualisante (ibid.) permet la formation du Moi au sein du Soi et celle d'un sentiment d'individualité grâce à ce qui est renvoyé en écho à l'enfant par le miroir visuel et sonore du visage et des gestes de la mère. L'enveloppe transitionnelle paradoxale (ibid.) assure à la fois la séparation et l'union de la peau de la mère et de la peau de l'enfant. Enfin, l'enveloppe tutélaire (ibid.) est à l'origine de l'acquisition du sentiment de continuité de soi.

Dans un troisième temps nous avons repéré les enveloppes impliquées dans le système perception/ conscience. L'enveloppe d'excitation (Anzieu, 1990) constitue le premier feuillet externe de l'enveloppe psychique qui remplit une fonction pare-excitatrice. L'enveloppe de communication (ibid.) est le second feuillet interne de l'enveloppe psychique qui permet de faire communiquer l'environnement extérieur et l'environnement intrapsychique. Les enveloppes Moi, Peau et Pensée (Anzieu, 1994) n'ont pas fait l'objet de définition spécifique.
Nous avons dégagé une quatrième catégorie d'enveloppes ayant trait à la forme de l'appareil psychique en tant que conteneur. L'enveloppe sac (Anzieu, 1993) se présentant comme un simple conteneur, sans feuillet différencié bien qu'elle puisse être trouée ou fragilisée; l'enveloppe bord (ibid.) qui permet de délimiter un territoire fixe, avec une grande malléabilité en fonction des évènements extérieurs et intrapsychiques; l'enveloppe interface (ibid.) met en contact deux réalités différenciées; l'enveloppe garde-frontière (ibid.) contrôle les passages de l'environnement extérieur à l'environnement intrapsychique; l'enveloppe sphère (ibid.) donne un volume au Moi. Enfin, la forme de l'appareil psychique en tant que conteneur est modelée par l'enveloppe spatiale (Anzieu, 1990), qui favorise l'émergence d'une conscience sensorielle, et l'enveloppe temporelle (ibid.), qui permet l'émergence d'une conscience rythmique.

Nous avons repéré également une enveloppe agissant dans les relations d'objet: l'enveloppe prénarrative (Stern, 1999), qui organise les compétences narratives del'enfant. Elle est décrite ainsi : «L'enveloppe prénarrative est une unité qui englobe toutes les formes d'expérience subjective organisée et qui, fait important, comprend des expériences de relation d'objets comme les identifications, les fusions, les selfobjects, les intériorisations, les identifications projectives, etc. En fait, la grande majorité des enveloppesprénarratives, d'intérêtcliniquenaturellement, concernent la relation d'objet» (ibid., p. 89).

Enfin, une dernière catégorie rassemble les enveloppes dans les pathologies psychiques. Celle qui nous intéresse est l'enveloppe psychique dans les psychoses, or les spécificités de sa composition n'ont été que peu abordées jusqu'à aujourd'hui (bien qu'Anzieu en 1985 évoque globalement sa «toxicité»). Dans des travaux précédents (Vollon et Gimenez, 2014), nous avons avancé l'hypothèse que l'enveloppe psychique du patient psychotique est poreuse. Nous nous sommes appuyés notamment sur les travaux de M. Klein (1946) concernant l'oscillation du Moi entre intégration et désintégration dans les tout premiers temps de la vie d'un individu, et les travaux de W.R. Bion (1967) sur la personnalité psychotique et non psychotique. Nous en sommes venus à la conclusion que le Moi en tant qu'enveloppe psychique chez le patient psychotique présente une diversité texturielle dont la notion de porosité permet de rendre compte. Le terme de porosité renvoie alors à des vides non interconnectés (correspondant aux 
béances induites par le morcellement du Moi), mais également à des zones «pleines » et quantitativement plus denses (correspondant aux zones du Moi en interaction avec le monde extérieur), capables de perception et de filtrage et témoignant de l'existence d'une personnalité non psychotique chez le patient.

La présentation de l'ensemble de ces éléments théoriques nous a permis de constater que le Moi, le Moi-peau, et plus globalement l'appareil psychique d'un sujet, pouvaient être appréhendés, dès les premiers écrits freudiens, comme un ensemble de feuillets, d'enveloppes d'instances séparatrices aux qualités et fonctions multiples. Nous avons pu constater que la notion d'enveloppe psychique constituait en réalité, au-delà d'une simple articulation, une véritable refonte de la notion de Moi-peau. Opérer un recensement des différentes modalités de cette enveloppe proposé dans la littérature peut paraître fastidieux. Il constitue pourtant un répertoire efficace auquel nous allons pouvoir nous référer dans l'analyse de nos extraits cliniques. Nous ne pensons pas que ces modalités s'opposent, nous préférons les envisager comme autant de «facettes », de fonctions, qu'une même enveloppe psychique peut revêtir selon la structuration psychique d'un individu, la pathologie qu'il présente, ses expériences vécues, etc. Nous allons pouvoir maintenant étudier dans quelle mesure ces modalités se mettent en scène dans un dispositif de groupe thérapeutique et quelles perspectives cliniques elles peuvent apporter au thérapeute.

\section{Extraits cliniques}

Le dispositif groupal dont sont extraites nos séquences est un groupe semi-fermé, composé de huit patients qui présentent une schizophrénie de type paranoïde et qui sont hospitalisés depuis au moins 1 an: Louis (56 ans), Pierre (53 ans), Adrien (32 ans), Abdel (49 ans), Judith (50 ans), Nathalie (43 ans), Jean (45 ans), Claude (52 ans). Chacun d'entre eux s'est engagé à venir régulièrement aux séances et à prévenir le groupe de toute absence éventuelle; le renouvellement ou l'arrêt de l'engagement s'effectue à la fin de l'année scolaire. Le groupe se réunit de façon hebdomadaire, pendant une heure. Deux cothérapeutes mènent le groupe, la psychologue du service et une infirmière. Les séances font l'objet d'un enregistrement sonore. Les règles y sont celles de l'association libre (chaque patient est invité à lais- ser venir ses pensées, ses sensations et ses émotions, et les traduire en mots), de la ponctualité, du respect de la parole de chacun, et de la discrétion des membres du groupe sur ce qui est dit. Ces règles sont énoncées au début de chacune des séances, tout nouveau participant devant s'engager préalablement à respecter ces règles pour pouvoir participer au groupe. Il s'agit ici des extraits de la sixième séance.

\section{L'enveloppe psychique comme prisme analytique $d u$ comportement individuel au sein du groupe}

Depuis le début de la séance, Nathalie cherche à prendre la parole. Lorsqu'elle y parvient, elle évoque une expérience vécue dans l'institution: «Il y a eu une marée haute, ils ont tout balayé. L'eau qui fuit, je ne sais pas d'où elle est sortie, cette eau, les femmes de ménage, elles nettoyaient le patio». Les cothérapeutes entendent cet énoncé dans sa dimension métaphorique à travers un double scénario: «quelque chose fuit» et «quelqu'un répare quelque chose». La psychologue invite le groupe à dire ce qui vient à partir de ces scénarios. Nathalie associe: «Ma grandmère, quand j'étais jeune, montait sur la table et nettoyait le lustre, elle avait un petit oiseau, tout mignon, tout beau, tout mignon, tout jaune, je lui donnais du pain. Il est mort.» Jean intervient, en reprenant sur les premiers scénarios: «Oui, il y a eu une fuite d'eau à l'étage, il a fallut qu'on descende pour que les femmes de ménage nettoient. » Nathalie complète alors: «Il y a eu une marée haute, ils ont tout balayé ».

A travers la référence à l'expérience d'un événement partagé par l'ensemble des membres du groupe (la fuite d'eau dans le pavillon), Nathalie parvient à mettre en lien l'événement de l'inondation au premier étage du pavillon commun aux membres du groupe avec des souvenirs personnels du passé. Ces souvenirs permettent d'aborder un certain nombre de thèmes pouvant être interprétés comme des éléments issus de son transfert sur le groupe: la figure maternelle (à travers la figure des femmes de ménage, du positionnement de la patiente envers sa poupée), la mort (avec le souvenir de l'oiseau, mais aussi cette marée haute qui balayerait tout sur son passage), les soins (par l'image de la grand-mère qui prend soin de son intérieur, de la patiente qui prend soin de sa poupée, des femmes de ménage qui nettoient et réparent les dégâts de l'inondation). Lorsque Natha- 
lie évoque la fuite d'eau, elle rapporte ainsi un élément perçu dans l'environnement extérieur, qu'elle parvient à mettre en lien avec des vécus internes, des souvenirs spécifiques: la fuite d'eau semble ici être un articulateur entre son passé, son investissement sur le groupe, et son quotidien à l'hôpital qu'elle partage également avec les autres membres du groupe. Relater cet événement lui permet par ailleurs d'instaurer un dialogue avec les autres membres du groupe: Jean s'appareille à sa chaîne associative individuelle en témoignant lui aussi de la fuite d'eau au premier étage du pavillon. Nous pensons que cette capacité de liaison est la manifestation de la fonction «interface» de son enveloppe psychique car, comme nous l'avons indiqué plus haut, elle permet de mettre en contact deux réalités différenciées, ici en l'occurrence: celle vécue par les membres du groupe au sein de l'institution et celle vécue à l'intérieur de chacun des membres du groupe.

Quelques minutes plus tard, Nathalie intervient de façon plus péremptoire pour évoquer les heures des repas et la composition des menus, laissant s'exprimer la partie plus psychotique de sa personnalité (Bion, 1967) : "C'est pas encore l'heure de manger, je ne sais pas ce qu'on mange ce midi, la dernière fois on s'est régalés parce qu'on avait de la choucroute» avant d'ajouter: "On a eu du couscous une fois, le vendredi on a du poisson ». Contre-transférentiellement, les cothérapeutes vivent cette intervention avec difficulté, ils se rendent compte qu'elle a un effet sidératif, tout se passant comme si elle venait attaquer leur propre appareil à penser ${ }^{3}$, faisant également émerger en eux une sensation de "polyphonie discordante» (Gimenez, 2006). Ils ressentent par ailleurs un certain agacement lié au caractère anecdotique et répétitif de cette intervention, ce qui se traduit par des fantasmes empreints d'agressivité à son égard: l'un d'entre eux ressent pendant la séance jusqu'à l'envie de la bâillonner pour ne plus l'entendre parler. Il est possible d'observer ici la manifestation de la défaillance de la fonction sac de l'enveloppe psychique (c'est-à-dire l'enveloppe comme conteneur de l'environnement interne) de Nathalie, mais également de la défaillance de la fonction gardefrontière de l'enveloppe psychique des co-thérapeutes (c'est-à-dire sa capacité filtrante). Tout semble se passer comme s'il n'existait plus de filtres permettant de résister mais également de contenir les mouvements destructeurs éprouvés par la patiente.
Une question se pose alors: comment expliquer la manifestation contradictoire de ces différentes fonctions pour un même patient au sein de cette séance? Que ce soit la fonction d'interface, la fonction sac ou la fonction garde-frontière, toutes appartiennent à la catégorie d'enveloppes ayant trait à la forme de l'appareil psychique en tant que conteneur (Kaës, 1976). Les thérapeutes se demandèrent alors si le groupe avait pu amener cette catégorie d'enveloppes à s'exprimer et pour quelles raisons? Comment accompagner la patiente dans cette expression? Comment border et atténuer progressivement ses manifestations contradictoires? Pourquoi cette mobilisation est-elle venue remettre en question la capacité de contenance de l'appareil psychique de chacun des thérapeutes? Nous nous rendons compte alors que l'analyse des enveloppes psychiques individuelles (celle de la patiente et celles des cothérapeutes) permet d'introduire de nouveaux questionnements non seulement sur le fonctionnement de Nathalie, mais également sur celui du groupe en général. C'est ce que nous allons montrer dans notre second extrait.

\section{Analyse de la texture de l'enveloppe psychique du groupe}

Un peu plus tôt dans la séance, Abdel arrive en retard et souhaite qu'on lui confirme la possibilité de ressortir s'il le souhaite: "Mais j'ai le droit de repartir pour aller aux toilettes, quand même?». Louis poursuit: «Je vous annonce, Messieurs Dames, que je vais partir à Paris, je voulais vous dire au revoir à tous et puis c'est bon. » Nathalie reprend, comme rapporté plus haut: «Il y a eu une marée haute, ils ont tout balayé. L'eau qui fuit, je ne sais pas d'où elle est sortie, cette eau, les femmes de ménage, elles nettoyaient le patio.»

La chaîne associative groupale semble ici rendre compte de l'émergence de scénarios de base (Gimenez, 2006) relatifs à des mouvements d'entrées et de sorties importants au sein du groupe. Nous empruntons le terme de "chaîne associative groupale » à Kaës (2010) qui la définit comme un tissu d'associations complexe issu du processus de concaténation, d'articulation de la chaîne associative groupale ainsi que des différentes chaînes associatives individuelles. Il est possible de mettre en lien l'émergence de ces mouvements d'entrées et de sorties (l'annonce du

\footnotetext{
$3 \quad$ Tel que peut le définir Bion, 1967.
} 
départ de Louis, la fuite d'eau dans le pavillon, le départ d'Abdel aux toilettes) avec l'expression de la défaillance de la fonction garde-frontière (Anzieu, 1993) de l'enveloppe psychique du groupe lui-même. Nous employons volontiers le terme d' "enveloppe psychique du groupe » puisque Anzieu (1985) a mis en évidence la possible d'envisager le groupe comme une enveloppe (il emploie alors le terme de Moi-peau groupal). Il peut donc être intéressant de mettre à profit le répertoire des modalités de l'enveloppe psychique présenté ci-dessous au service de la compréhension des événements qui se produisent à ce moment-là de la vie du groupe. Nous pouvons interpréter l'apparition de ces scénarios comme l'expression de l'inconsistance de l'enveloppe psychique groupale. Cette inconsistance peut s'expliquer notamment par la projection d'angoisses d'intrusion du côté d'Abdel et de Louis, qui tentent de préserver leurs individualités respectives face au groupe en envisageant la possibilité de s'en extraire (repartir aux toilettes pour Abdel et partir définitivement dans une autre ville pour Louis).

Dans un second mouvement, nous observons une évolution de cette inconsistance, ou plutôt l'expression d'une autre fonction de l'enveloppe psychique groupale. Claude témoigne de son vécu dans le pavillon: "Maintenant ici je suis bien, je suis bien entouré, le personnel c'est des professionnels. Si j'ai un coup de blues il y a toujours quelqu'un pour parler avec moi, ça ça me fait chaud au cœur et je vous remercie tous. » Louis et Jean poursuivent en verbalisant leur reconnaissance envers la présence et l'empathie dont le personnel soignant peut faire preuve, et Nathalie conclut: "On descend les chaises pour midi, ou sinon quand les femmes de ménage, l'après-midi, elles font le ménage, moi je regarde la télévision, je chante, je danse.» Contre-transférentiellement, les cothérapeutes se surprennent à ressentir un sentiment de bien-être et de sécurité intense. Par l'émergence de l'illusion groupale (Anzieu, 1971) portant sur le comportement «bienveillant» des soignants du pavillon vis-à-vis des patients, il est intéressant de constater que les angoisses d'intrusion et la défaillance de la fonction garde-frontière de l'enveloppe psychique groupale ont laissé place à des mouvements psychiques plus sécurisants relatifs à l'expression possible de sa fonction individualisante. Cette fonction permet la formation d'un Moi groupal: c'est-à-dire une structuration psychique groupale, partagée par tous, qui permet l'émergence d'un sentiment de contenance et de maintenance psychique.

\section{En guise de conclusion}

Notre démarche avait ici pour objectif de tenter d'apporter quelques précisions sur les notions de Moipeau et d'enveloppe psychique, d'en dégager si possible une certaine heuristicité dans la pratique clinique. Nos rappels théoriques étaient nécessaires pour deux raisons. Ils ont permis, dans un premier temps, de mettre en exergue que la notion d'enveloppe psychique était une refonte de la notion de Moi-peau. Dans un second temps, ils ont rendu compte des modalités multiples de cette enveloppe, nous conduisant à proposer l'ébauche d'un répertoire des différentes fonctions qu'elle peut assumer au sein de l'appareil psychique. En nous saisissant de ce répertoire pour analyser les interventions des patients du groupe de parole, nous avons pu dégager des interrogations cliniques, mais surtout envisager la mobilisation de certaines fonctions de l'enveloppe psychique individuelle en situation de groupe, autrement impensée si nous n'y avions pas eu recours. Enfin, nous souhaitons nous concentrer maintenant sur le dernier extrait présenté. Par la référence aux différentes fonctions de l'enveloppe psychique, nous avons pu apprécier que ce ne furent pas les mêmes modalités de l'enveloppe psychique du groupe qui étaient mobilisées tout au long de la séance. D'une texture inconsistante mise en évidence par une défaillance de la fonction pare-excitante de l'enveloppe du groupe, elle a évolué vers une texture plus consistante exprimée via la fonction individualisante de l'enveloppe. Nous pouvons apprécier la modification de cette texture, nous donnant accès aux potentiels processus transformationnels en jeu dans les groupes thérapeutiques pour les patients psychotiques particulièrement chroniques. En effet, nous pensons que le groupe évolue dans son organisation (par exemple: nature des mouvements transférentiels, positionnement psychique des thérapeutes...) et sa structuration (par exemple: qualité de l'enveloppe psychique groupale, aménagement de réseaux de communications spécifiques...) au fur et à mesure des séances. Il semble alors nécessaire d'avoir recourt à des outils qui lui permettent d'avoir accès à ces transformations pour mieux en saisir les enjeux cliniques et thérapeutiques. 
Summary

This article's objective is to interrogate and redefine the concepts of skin-ego and psychic envelope. It is a preliminary stage in view of a more global study on the quality and dynamics of psychic envelopes, be it when working in individual psychotherapy or in group therapy. Our method consists in reviewing various epistemological contributions, notably those from the Freudian corpus to throw a different perspective on the concept of skin-ego and psychic envelopes which in the literature maintain a strong interdependance. This approach will enable us gradually to observe that there are several types of skin-ego but also a multitude of psychic envelopes that we propose to identify here for the first time and organize in six specific categories.

\section{Bibliographie}

Anzieu D. (1971): L'illusion groupale, in: Le groupe et l'inconscient. Paris, Dunod, 1984, pp.??-??.

Anzieu D. (1985): Le Moi-peau. Paris, Dunod.

Anzieu D. (1987): Les enveloppes psychiques. Paris, Dunod. Anzieu D. (1990): L'épiderme nomade et la peau psychique. Paris, Les Editions du Collège de Psychanalyse Groupale et Familiale. Anzieu D. (1993): Les contenants de pensée. Paris, Dunod. Anzieu D. (1994) : Du Moi-peau au Moi-pensant. Paris, Dunod. Bion W.R. (1967): Réflexion faite. Paris, PUF, 2007.

Brazelton T. B. (1982) : La dynamique du nourrisson. Paris, ESF.

Falgiere J. (2002) : L'élaboration d'une pratique et sa théorisation, in: Falguière J. (dir.) : Analyse de groupe et psychodrame. Fondements théoriques, dispositifs et pratiques en institution. Ramonville-St-Agne, Erès, pp. 29-77.

Freud S. (1895): Esquisse d'une psychologie scientifique, in : Introduction de la psychanalyse. Paris, PUF, 2009, pp. 130-156.

Freud S. (1900): L'interprétation des rêves. Paris, PUF, 2007.

Freud S. (1914): Pour introduire le narcissisme, in : La vie sexuelle. Paris, PUF, 2009, pp. 80-105.

Freud S. (1923): Le Moi et le Ça. Paris, PUF, 2009.

Freud S. (1925): Notes sur le «bloc-notes magique », in: Résultats, idées, problèmes II. Paris, PUF, 2007, pp. 120-124.

Gimenez G. (2006) : La construction d'une chaîne associative groupale dans le travail psychanalytique avec les patients psycho- tiques en groupe: du chaos dissociant à une possible polyphonie. Rev. Psychothér. Psychanal. de Groupe, 2 (47): 79-91.

Houzel D. (1994) : L'enveloppe psychique, métaphore et processus, in: Les voix de la psyché. Hommage à Didier Anzieu. Paris, Dunod, 2000, pp. 164-190.

Kaës R. (1976) : L'appareil psychique groupal. Paris, Dunod.

Kaës R. (1979) : Crise, rupture et dépassement. Paris, Dunod, rééd. 2004.

Kaës R. (2007): Du Moi-peau aux enveloppes psychiques. Genèse et développement d'un concept. Le Carnet PSY, 117: 33-39.

Klein M. (1946) : Notes sur les mécanismes schizo-paranoïdes, in : Développements de la psychanalyse. Paris, PUF, 1980, pp.??-??

Laugier F., Toliou A. (2009): Penser les soins spécifiques des patients psychotiques institutionnalisés au long cours. Psychothérapies, 29 (3): 175-186.

Stern D. (1999): L'enveloppe pré-narrative, in: Narration et psychanalyse. Paris, l'Harmattan, pp. 102-120.

Vollon C., Gimenez G., Bonnet C. ( 2014): Mise en groupe, psychoses et porosité des enveloppes psychiques. Rev, Psychother., Psychanal. de Groupe, 64 (3) : 203-214. 